\title{
Childhood onset SLE- case report
}

\begin{abstract}
Systemic lupus erythematosus (SLE) is a chronic autoimmune disease that can affect any organ and may result in significant morbidity and mortality. Childhood-onset c-SLE is a rare disease with an incidence of 0.3-0.9/100,000 children-years and a prevalence of 3.38.8/100,000 children. ${ }^{1}$ Asians, African American, Hispanic and Native Americans have higher frequency of the disease. ${ }^{2,3}$ Similar to adult SLE, c-SLE affects mainly females with around $80 \%$ of the affected children. According to the literature, the median age of onset is 11-12 years and it is rare in children under 5 years of age. The manifestations of c-SLE are variable and most of the times are atypical compared to adult SLE which make the diagnosis of cSLE a challenge to the treating physician. ${ }^{4}$ This report shows a series of presentation in pediatric patient who finally was diagnosed with SLE.
\end{abstract}

Volume II Issue 2 - 202 I

\author{
Awal Alhusain,' Hasan Alhashim, ${ }^{2}$ Wafa \\ Almuraidif, ${ }^{2}$ Mariam Alghazal ${ }^{2}$ \\ 'Dammam Regional Lab and Blood Bank, Saudi Arabia \\ ${ }^{2}$ Maternity and Children Hospital Dammam, Saudi Arabia
}

Correspondence: Alhusain A, Dammam Regional Lab and Blood Bank, Saudi Arabia, Email aalmohmedhusain@moh.gov.org

Received: January 19, 2021 | Published: August 04, 2021

Keywords: Pediatric, childhood, SLE, pulmonary, pneumonitis, chest infection, nephritis, lupus

\section{Case report}

A 7 year old Saudi female patient presented to the Maternity and Children Hospital $(\mathrm{MCH})$ with a history of high grade fever, productive cough and hemoptysis. The patient gave history of subjective weight loss and fatigability. There was no history of loss of appetite, GI symptoms, urinary tract symptoms or history of joint pain or swelling. There was a history of travel to India five months prior to her presentation. The patient's symptoms started on the last day of her trip. The patient was treated in many hospitals with several courses of antibiotics and the impression was URTI. However, the patient has no or minimal improvement. Two weeks before presenting to the hospital, she was admitted for RT sided lobar pneumonia.

On examination, the patient looks pale, not cyanosed, of average built, coughing frequently during examination. She weighs: $22.5 \mathrm{~kg}$, height: $123 \mathrm{~cm}$, febrile: 38.7 , with RR: 34 B/m, HR: 138, BP: 108/64. She had grade 1-2 finger clubbing, and reduced air entry on the left chest and bilateral crackles.

The impression was:

- Pulmonary TB

- Cystic fibrosis

- Primary immunodeficiency disease.

In a period of three months, the patient was admitted three times and managed with antibiotics.

She was investigated with the following results:

CBC showed anemia with $\mathrm{Hb}: 9.4 \mathrm{~g} / \mathrm{dl}, \mathrm{MCV}$ : 68.1, MCH, lymphopenia with lymphocyte count $2.1 \%$.

Screening for TB was negative

Blood culture and sputum culture were negative.

Renal profile, serum electrolyte, LFT were normal

\section{High resolution $\mathrm{CT}$}

The patient was referred to immunology; immunophenotyping revealed lymphopenia of all lymphocyte subsets. Immunoglobulins showed marked increase of IgG (3900) normal range (400-1400).

\section{Autoimmune profile}

ANA $>1: 1280$, DsDNA 1:320, with low complement level.

Renal biopsy revealed lupus nephritis INS/RPS class IV-G (A) with ANCA released vasculitis.

Finally, the patient was diagnosed with SLE and started on prednisolone treatment with good improvement.

\section{Discussion}

SLE is an autoimmune disease associated with the formation of autoantibodies against self-antigens. These autoantibodies are associated with inflammatory tissue damage of the affected organ/ tissue. Although SLE affects mainly women of the child-bearing age, the incidence of childhood SLE may represent $10-20 \%$ of SLE patients. ${ }^{5-7}$

The mean age of onset in childhood SLE is between 11-12 years ${ }^{5,6}$ and more prevalent in Asians, Hispanic, and Caucasians girls. ${ }^{7}$ According to the literature, the most presenting symptoms include fever, nephropathy, and lymphadenopathy. ${ }^{6}$ Children usually have more active disease and thus need more intensive treatment compared to adults.

Pleuro-pulmonary manifestation occurs in around $50-70 \%$ of adults with SLE ${ }^{8,9}$ In children, the pulmonary involvement in SLE is variable due to different study designs. The majority of studies reported $5-30 \%$ of children with SLE having pulmonary manifestations. Most of them have asymptomatic pulmonary involvement diagnosed with pulmonary function test (PFT) or high resolution CT scanning. Clinical pulmonary involvement, although rare, include pulmonary hypertension, diffuse interstitial lung disease, pulmonary hemorrhage, acute lupus pneumonitis and pneumothorax. ${ }^{10-12}$ On the other hand, pleuro-pulmonary infection and subclinical lung disease are more frequent and may proceed the diagnosis of SLE similar to the presentation of this particular patient. ${ }^{13}$

In c-SLE renal involvement occurs in up to $75 \%$ of the affected children, $90 \%$ of those will develop renal disease within the first two years after diagnosis. ${ }^{14}$ The clinical presentation ranges from minimal proteinuria and microscopic hematuria to nephrotic-range proteinuria, urinary casts, severe hypertension and renal insufficiency or renal 
failure. SLE affects mainly the glomerulus (lupus nephritis) and rarely affects the interstitium. The severity of lupus nephritis does not correlate with the severity of clinical presentation, thus a renal biopsy and histologic examination must be performed. As in our patient, the disease period was around five months, yet she had grade IV lupus nephritis. This lesion is the most frequent and with more than $80 \%$ of c-SLE biopsies demonstrating this lesion. ${ }^{7,14}$

Patients with these features are at high risk of having end stage renal disease (ESRD) and intensive treatment is mandated. All patients with SLE should have regular checkup for blood pressure, serum creatinine, and urinalysis for proteinuria, hematuria and evidence of urine casts. The incidence of ESRD had dropped in the past decades due to the use of aggressive treatment. ${ }^{7}$

Laboratory testing can support the diagnosis of SLE in the presence of suggestive symptoms. It is well documented that SLE is characterized by the production of multiple autoantibodies. The most common of which is ANA which present in around $95 \%$ of cSLE patients. In addition, anti dsDNA and the extractable nuclear antigens (ENA) which are more specific for SLE and correlate more with disease feature.

In this particular patients, the symptoms were vague and the laboratory testing was the first clue for suspecting SLE. We found high IgG level and that gave the suspect of having autoimmune disease. When we performed further testing, we found positive ANA $>1: 1280$, positive anti dsDNA 1:320 and low complement C3 level. These findings along with lymphopenia support our suspicion of autoimmune disease SLE. The patient has fulfilled four out of the 11 ACR classification criteria for SLE $\dot{2}^{15}$ positive ANA, positive DNA, lymphopenia and fever as one of the constitutional symptoms.

Later on, the patient developed symptoms of renal involvement and kidney biopsy further confirmed the diagnosis. Routine hematology and biochemistry tests are recommended for the follow up of cSLE and to monitor for flare versus remission, drugs side effects, and the effects of chronic inflammation. Urinalysis is mandatory in general as renal impairment is one of the features of disease course. Patients with renal involvement should be tested for urine protein/creatinine ratios (spot or 24 hour collection) for monitoring the response to treatment of lupus nephritis. SLE is called the great mimicker thus its diagnosis is made by exclusion. It shares clinical features with multiple diseases such as infection, malignancy, immunodeficiency and other diseases. Adults patients present with photosensitivity, malar rash, Raynaud's phenomenon, or pleural effusion are not challenging for diagnosis. However, pediatric patients present with vague symptoms such as fever, weight loss, fatigue, anemia, leukopenia, thrombocytopenia, arthralgia or arthritis which are common for diseases that have systemic features and thus make the diagnosis of cSLE a challenge for treating physicians.

\section{Conclusion}

cSLE is a rare but severe autoimmune disease with multiple organ involvement and has broad range of disease manifestations. The diagnosis of cSLE is a great challenge, however early diagnosis is important to prevent adverse outcomes and organ damage. SLE in children has more severe form with higher incidence of morbidity and mortality compared with adult onset SLE. It should be considered that this disease is lifelong, unpredictable and has a relapse-remission nature.

\section{Acknowledgments}

None.

\section{Conflicts of interest}

None.

\section{References}

1. Kamphuis S, Silverman ED. Prevalence and burden of pediatric-onset systemic lupus erythematosus. Nat Rev Rheumatol. 2010;6:538-546.

2. Hiraki LT, Benseler SM, Tyrrell PN, et al. Ethnic differences in pediatric systemic lupus erythematosus. J Rheumatol. 2009;36:2539-2546.

3. Pons-Estel GJ, Alarcon GS, Scofield L, et al. Understanding the epidemiology and progression of systemic lupus erythematosus. Semin Arthritis Rheum. 2009;39:257-268.

4. Segel M, Lee SL. The epidemiology of systemic lupus erythematosus Semin Arthritis Rheum. 1973;3:154

5. Meislin AG, Rothfield N. "Systemic lupus erythematosus in childhood. Analysis of 42 cases, with comparative data on 200 adult cases followed concurrently." Pediatrics. 1968;42:37-49.

6. Font J, Cervera R, Espinosa G et al. "Systemic lupus erythematosus (SLE) in childhood: analysis of clinical and immunological findings in 34 patients and comparison with SLE characteristics in adults." Annals of the Rheumatic Diseases. 1998;57:456-459.

7. Levy DM, Kamphuis S. "Systemic lupus erythematosus in children and adolescents." Pediatric Clinics of North America. 2012;59:345-364.

8. Haupt HM, Moore GW, Hutchins GM. The lung in systemic lupus erythematosus. Analysis of the pathologic changes in 120 patients. Am J Med. 1981;71:791-798.

9. Hunninghake GW, Fauci AS. Pulmonary involvement in collagen vascular diseases. Am Rev Resp Dis. 1979;119:471-503.

10. Caeiro FM, Michielson FMC, Bernstein R, et al. Systemic lupus erythematosus in childhood. Ann Rheum Dis. 1981;40:325-331.

11. De Jongste JC, Neijens HJ, Duiverman EJ, et al. Respiratory tract disease in systemic lupus erythematosus. Arch Dis Child. 1986;61:478-483.

12. Delgado EA, Malleson PN, Pirie GE, et al. The pulmonary manifestations of childhood onset systemic lupus erythematosus. Semin Arthritis Rheum. 1990;19:285-293.

13. Cerveri I, Bruschi C, Ravelli A, et al. Pulmonary function in childhood connective tissue diseases. Eur Respir J. 1992;5:733-738.

14. Hiraki LT, Benseler SM, Tyrrell PN, et al. Clinical and laboratory characteristics and long-term outcome of pediatric systemic lupus erythematosus: a longitudinal study. J Pediatr. 2008;152:550-556.

15. Hochberg MC. Updating the American College of Rheumatology revised criteria for the classification of systemic lupus erythematosus. Arthritis Rheum. 1997:40:1725. 